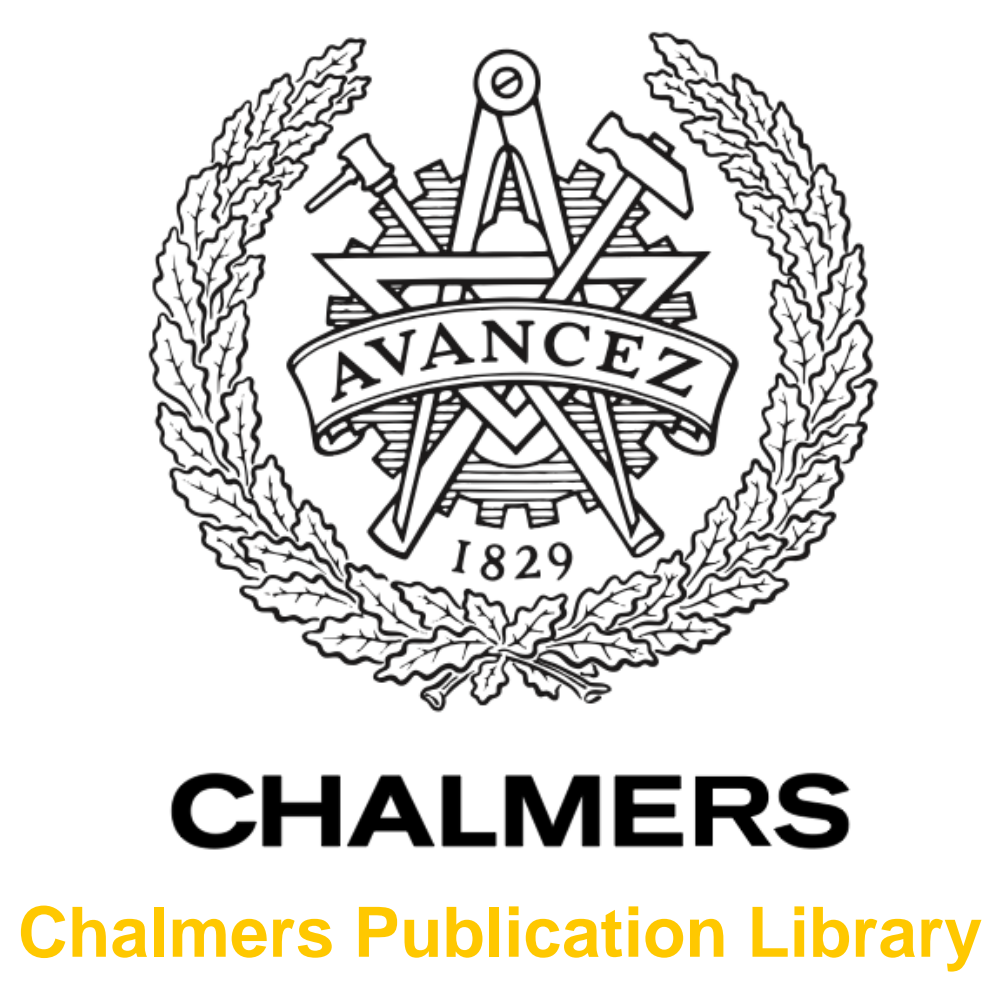

\title{
Development of a detailed system model of the Eleven feed receiver using the CAESAR software
}

This document has been downloaded from Chalmers Publication Library (CPL). It is the author's version of a work that was accepted for publication in:

Proceedings of 6th European Conference on Antennas and Propagation, EuCAP 2012. Prague, 26-30 March 2012

Citation for the published paper:

Klein, B. ; Ivashina, M. ; Maaskant, R. (2012) "Development of a detailed system model of the Eleven feed receiver using the CAESAR software". Proceedings of 6th European Conference on Antennas and Propagation, EuCAP 2012. Prague, 26-30 March 2012 pp. 3416-3420.

http://dx.doi.org/10.1109/EuCAP.2012.6206386

Downloaded from: http://publications.lib.chalmers.se/publication/156672

Notice: Changes introduced as a result of publishing processes such as copy-editing and formatting may not be reflected in this document. For a definitive version of this work, please refer to the published source. Please note that access to the published version might require a subscription. 


\title{
Development of a Detailed System Model of the Eleven Feed Receiver Using the CAESAR Software
}

\author{
Benjamin Klein*, Marianna V. Ivashina ${ }^{\dagger}$, Rob Maaskant ${ }^{\dagger}$, Alan R Clark*, and Miroslav Panteleev ${ }^{\ddagger}$ \\ * School of Electrical and Information Engineering, Wits University, Johannesburg, South Africa, Email: ben@hartrao.ac.za \\ † Chalmers University of Technology, S-41296 Gothenburg, Sweden, Emails: ivashina@chalmers.se, maaskant@chalmers.se \\ $\ddagger$ Onsala Space Observatory, SE-439 92 Onsala, Sweden, Email: pantaleev@ chalmers.se
}

\begin{abstract}
A mathematically general method is presented for exporting the far-field patterns, radiation efficiency and impedance data, for multi-port antennas, from EM solvers to the microwave circuit simulator of the CAESAR software in order to perform the analysis of a multi-port antenna-receiver system. This hybrid modelling approach offers a few important advantages over the early developed modelling methods and standard simulation tools; including the capability to predict the correlated noise contributions due to both internal and external noise sources, while exploiting the translation/rotation symmetry of the antenna structure to reduce computation time. This approach is validated using folded dipole antennas and applied to model a more complex antenna-receiver system the Eleven antenna feed operating from 2 to $12 \mathbf{G H z}$ which consists of four log-periodic dipole arrays co-integrated with LNAs, a balun and hybrid combining network. The Eleven feed receiver model is verified by comparing the simulation results with the measurements performed with a practical system.
\end{abstract}

\section{INTRODUCTION}

Next generation radio astronomy systems and Earth observation science rely largely on innovative antenna technologies (such as wideband dense arrays and multi-port feeds for reflector antennas) and low-noise receivers. Examples of on-going developments are the SKA radio telescope and VLBI2012 network [1], [2]. Design and optimization of such highsensitivity wide-band systems is challenging, due to the computationally intensive nature of the antenna simulations (over wide frequency and scan angle ranges), and strong interaction between antenna element mutual coupling, receiver noise and beam forming scenarios [3]. To tackle these problems, a number of computationally efficient numerical approaches, combined antenna-receiver characterization techniques and dedicated software tools have recently been developed [4]-[6]. One example is the CAESAR software, an advanced EM-MW solver for the analysis of large antenna array systems [4], and a newly developed phased-array feed toolbox for the analysis of reflector antennas [6]. This software is tailored to solve for the noise response of receiver/antenna systems to external (ground and sky) and internal (LNAs, ohmic losses) noise sources. The necessary input parameters for the system noise performance calculation are: (i) antenna radiation patterns and radiation efficiencies and the antenna input impedance matrix; (ii) noise and $S$-parameters of LNAs and beam forming network; (iii) the external noise temperature distribution.
The goal of this study is to develop a detailed system model of the multi-port Eleven feed for a reflector antenna [7], and to perform the system noise analysis.

Problem formulation: The radiation patterns, efficiency and impedance of the Eleven feed are simulated in CST and can be exported to CAESAR to construct the overall system model. The excitation conditions for antenna ports and formats of the simulation data of the radiated field as implemented in CST and CAESAR are however different. Our objective is therefore to develop a general procedure for calculating the necessary data, for noise evaluation, from CST (or similar EM software), into the format required for modelling the system noise temperature in CAESAR.

\section{ARBITRARILY LOADED CASE}

\section{A. Model description}

In this section, the method to convert the radiation characteristics simulated for the source excitation and loading situation "a" to that of situation "b" is described, as illustrated in Fig. 1,

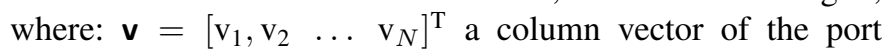
voltages (where $\mathrm{T}$ is the transposition operator), $\mathbf{i}$, similarly defined, are the antenna input port currents, $f$ is the total beam pattern defined as $\boldsymbol{f}(\theta, \phi)=E_{\theta}(\theta, \phi) \hat{\boldsymbol{\theta}}+E_{\phi}(\theta, \phi) \hat{\boldsymbol{\phi}}, \mathbf{Z}$ is the antenna $Z$-matrix, and $\mathbf{Z}_{L}$ is a diagonal matrix of the load impedances.

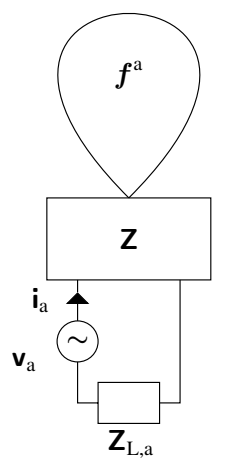

(a) Original situation "a"

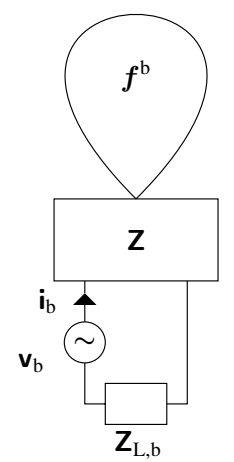

(b) Desired situation "b"
Fig. 1. Compact representation of a multi-port antenna, for the two cases, "a" and "b".

To completely characterise an $N$-port antenna device in the transmitting case, $M$ linearly independent voltage vectors, 
corresponding to $M$ antenna beam patterns, are required, where $M \geq N$. We define the column and row-augmented matrices:

$$
\begin{aligned}
\mathbf{V} & =\left[\begin{array}{l:l:l:l}
\mathbf{v}_{1} & \mathbf{v}_{2} & \ldots & \mathbf{v}_{M}
\end{array}\right] \\
\mathbf{f} & =\left[\begin{array}{l:l:lll}
\boldsymbol{f}_{1} & \boldsymbol{f}_{2} & \ldots & \boldsymbol{f}_{M}
\end{array}\right]^{\mathrm{T}}
\end{aligned}
$$

where $\mathbf{V}$ is a rectangular $N \times M$ matrix and $\mathbf{f}$ is a $M \times 2$ matrix (two field components). To calculate the required beam patterns, a new excitation voltage vector is required in "a" to produce the desired port voltage excitation in " $b$ ". This can be done using the equivalent circuit in Fig. 2, where the required port voltages to produce the required currents are defined as $\mathbf{v}_{\mathrm{a}}^{\prime}$. Solving for the desired current $\mathbf{i}_{\mathrm{b}}$, and using that the voltage

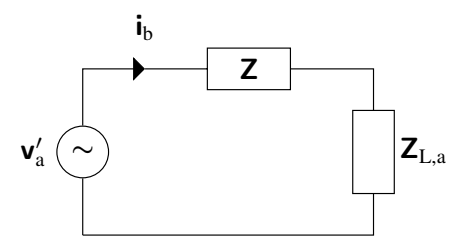

Fig. 2. Equivalent voltage excitation for case " $a$ " to realise current $\mathbf{i}_{\mathrm{b}}$ in situation "b".

linearly scales the fields, the final conversion equation is found to be:

$$
\mathbf{f}^{\mathrm{b}}=\mathbf{V}_{\mathrm{b}}^{\mathrm{T}}\left(\mathbf{Z}_{\mathrm{L}, \mathrm{a}}+\mathbf{Z}\right)\left(\mathbf{Z}_{\mathrm{L}, \mathrm{b}}+\mathbf{Z}\right)^{-1} \mathbf{V}_{\mathrm{a}}^{-\mathrm{T}} \mathbf{f}^{\mathrm{a}} .
$$

\section{B. Folded dipole example}

Eq. (2) is general, and can be applied for any new loading and excitation " $b$ ", as long as the original situation "a" is fully characterised. The conversion is validated for a simple case a folded dipole - simulated in both CST and CAESAR. The beam patterns simulated in CST are transformed using Eq. (2) and compared to the simulated antenna beam patterns from CAESAR. The original and converted patterns are plotted in Fig. 3. The slight differences (at low field levels) between the converted CST and CAESAR patterns are close enough to be within the expected variations due to using different EM simulators and meshed geometries.

\section{Modelling OF MULTi-PORT ANTENNA PATterns}

\section{A. Description of the Eleven antenna system}

The Eleven antenna is a decade-bandwidth log-periodic dual-dipole array developed at Chalmers University of Technology [7], consisting of eight single-ended ports connected to LNAs and a combining network as show in Fig. 4. The noise performance of this receiver has previously been analysed with an approximate model (based on the equivalent system representation [5], [7]) and assuming identical LNAs (with no reflections at their ports) and an ideal combiner. The CAESAR model, presented here, is a full numerical approach which can be applied to perform a detailed study of the Eleven feed performance and uses (where available) the measured noise and scattering parameters of LNAs and combiner components.

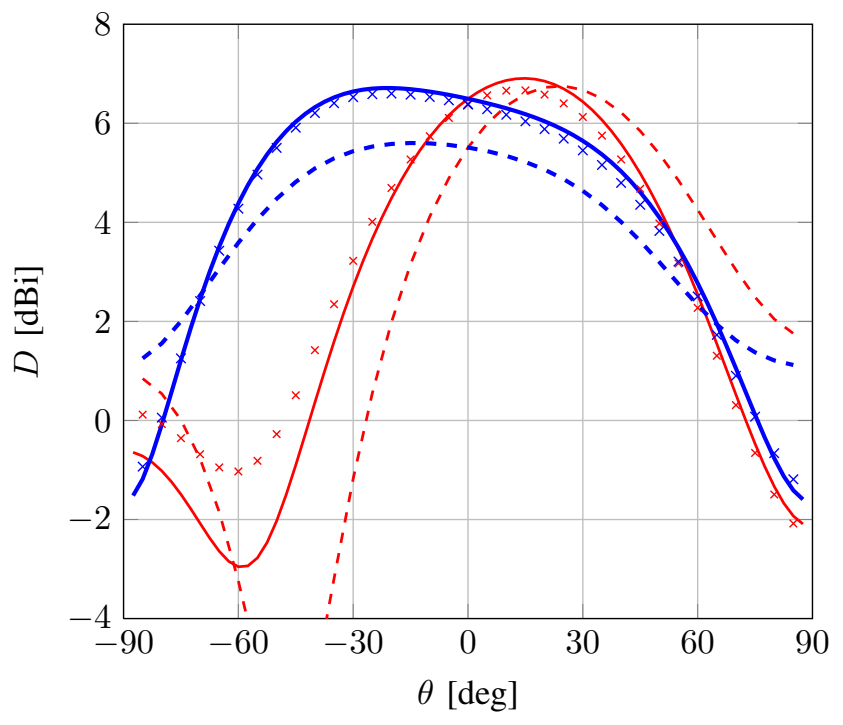

$-\phi=0^{\circ} \mathrm{CAE} \times \phi=0^{\circ} \mathrm{CST}$ conv. - - $\phi=0^{\circ} \mathrm{CST}$ $\longrightarrow \phi=90^{\circ} \mathrm{CAE} \times \phi=90^{\circ} \mathrm{CST}$ conv. - - $\phi=90^{\circ} \mathrm{CST}$

Fig. 3. Comparison between original CAESAR and converted CST beam patterns for a simple folded dipole at $5 \mathrm{GHz}$, two cross cuts are shown.

The numerical results are compared to measurements carried out with the practical system.

The Eleven antenna is a symmetric structure, and thus knowing the required combination network for the desired antenna beam pattern, means the simulation is typically done for only one eighth of the antenna using appropriate symmetry planes and associated boundary conditions for the fields [8]. Receiver noise, however, is coupled through all pattern correlations and so the CAESAR model requires the feed beam pattern to be modelled. From the inversion requirement in (2), eight ports require eight unique excitation cases to be able to produce the case for any arbitrary excitation and port termination. This is impractical over the wide bandwidth of the Eleven antenna and so a single port is simulated instead, with all other ports terminated, and then the simulated beam pattern is mathematically mirrored to produce the beam patterns for the equivalent single port excitations. This requires the full antenna to be simulated, but only for one excitation. It is pointed out, that it is computationally faster to simulate multiple smaller problems with varying $E$ and $H$ plane symmetries, as the number of mesh cells grows faster with a larger geometry than with the number of cases, however, the single port pattern is chosen in this case as it is easier to verify the implemented code with a simple test cases, as discussed below.

\section{B. Exploiting the rotation/translation symmetry}

The mirroring is done using $X, Y$ plane symmetries, and is tested using four simple folded dipoles configured to match the port configuration of the Eleven feed, but simulated at one frequency (so all ports and combinations can be quickly 


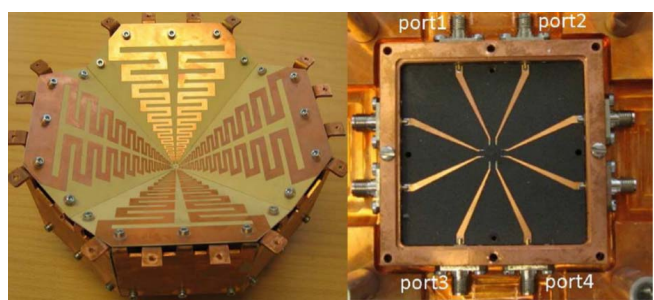

(a) Photos of the Eleven antenna consisting of four logperiodic dipole arrays and the descrambling board, realizing a transition to eight single-ended coaxial ports (on the rear side of the ground plane

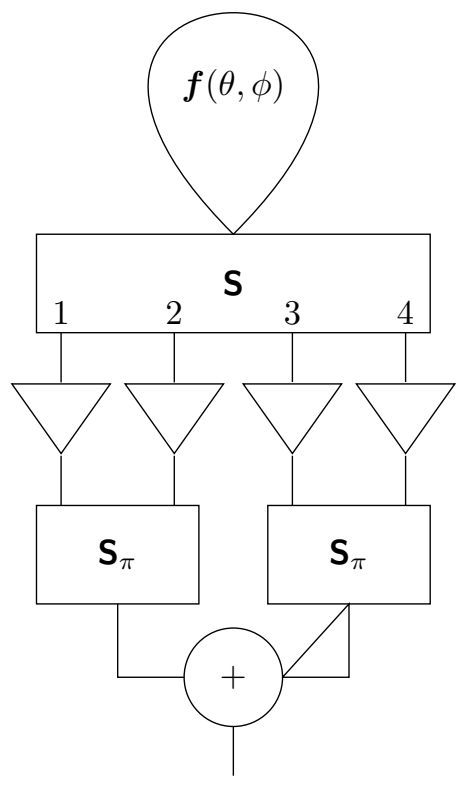

(b) The Eleven antenna receiver model, for one polarization, including two oppositely located dipole arrays, four single ended LNAs connected to the coaxial ports, two hybrids and combiner. The output ports of the other four LNAs are matched terminated.

Fig. 4. Photos of the Eleven antenna, and the overall system model

simulated and compared to the mirrored patterns), shown in Figs. 5, 6 and 7. The mirroring function shows only small variations $\left(5-10^{\circ}\right)$ in the $\phi=90^{\circ}$ plane. This accounts for similar variations in the summed patterns of the Eleven antenna case shown in Fig. 8. Further verification is done by comparing the appropriately summed single port beam patterns with the previously simulated total antenna beam pattern used to characterise the feed. The comparison is plotted (at a single frequency for brevity) in Fig. 8. The patterns are normalised and agree well with full port simulation (patterns within the simulated band, 1-12 GHz, show similar agreement). The small differences between the results are primarily due to the fact that the two simulations are performed in CST; one for the entire Eleven feed exciting a single port only, and another one for for one eighth of the antenna using symmetry planes
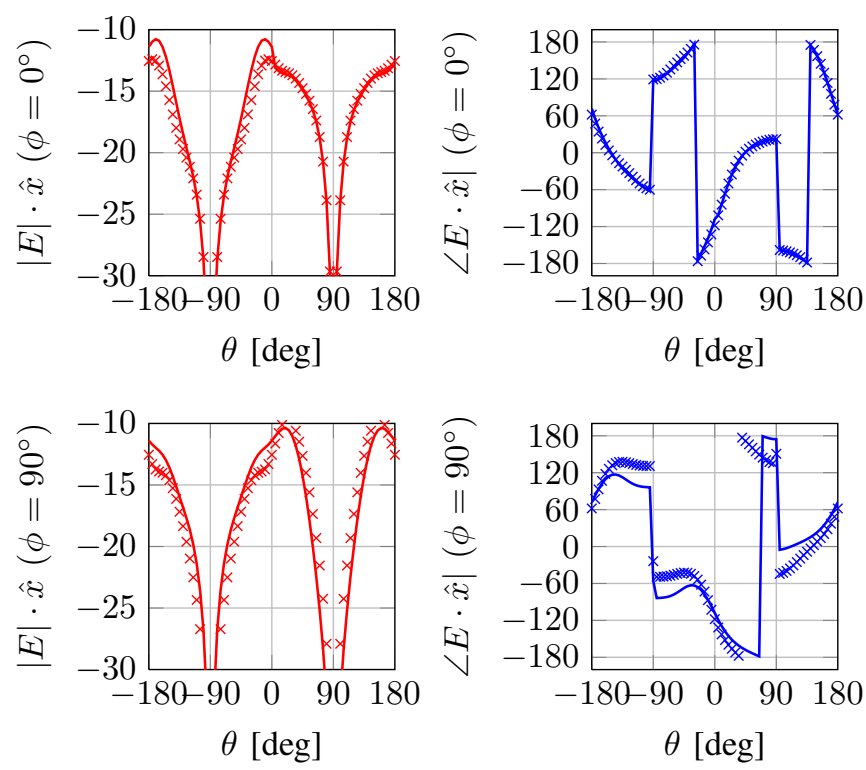

- Mirrored $\times$ Computed

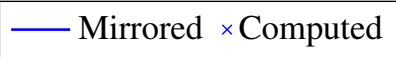

Fig. 5. Comparisons between mirrored and directly computed beam pattern cuts for port 2 of four folded dipoles arranged in an identical port layout as shown in Fig. 4, simulated at $5 \mathrm{GHz}$

for the fields.

\section{SYSTEM NOISE ANALYSIS}

The beam patterns are transformed through (2), and used directly in the CAESAR software. The method used is to compute the system gain, using a $1 \mathrm{~K}$ sky noise temperature distribution and an otherwise noiseless system. The noise temperature of the noisy system is then modelled and normalised by dividing out the system available noise gain. Before doing so an ideal system (matched, lossless and reflection less beam former) was modelled in CAESAR, and compared to simpler modelling predictions.

\section{A. Simpler Noise characterization models}

An ideal hybrid and combiner network, with cryogenic wideband Caltech LNAs with an Eleven feed can be modelled using a simple model assuming a perfect beamformer that can be approximated as a single channel with only one amplifier modelled using the standard Pospieszalski model [9], or more accurately using an equivalent system representation [5]. The equivalent model assumes no isolation and reflection losses in the LNAs, hybrids and combiner. The comparison is plotted below in Fig. 9. The variations between the CAESAR and the ideal model are due to approximation of the beam pattern which introduce small errors in the normalisation.

\section{B. Numerical and experimental results}

A single polarization of the Eleven feed is modelled assuming a $7 \mathrm{~K}$ atmospheric contribution and $300 \mathrm{~K}$ ground contribution with and a $15^{\circ}$ tree line. The model accounts for; external sky and ground noise sources, ohmic losses in 

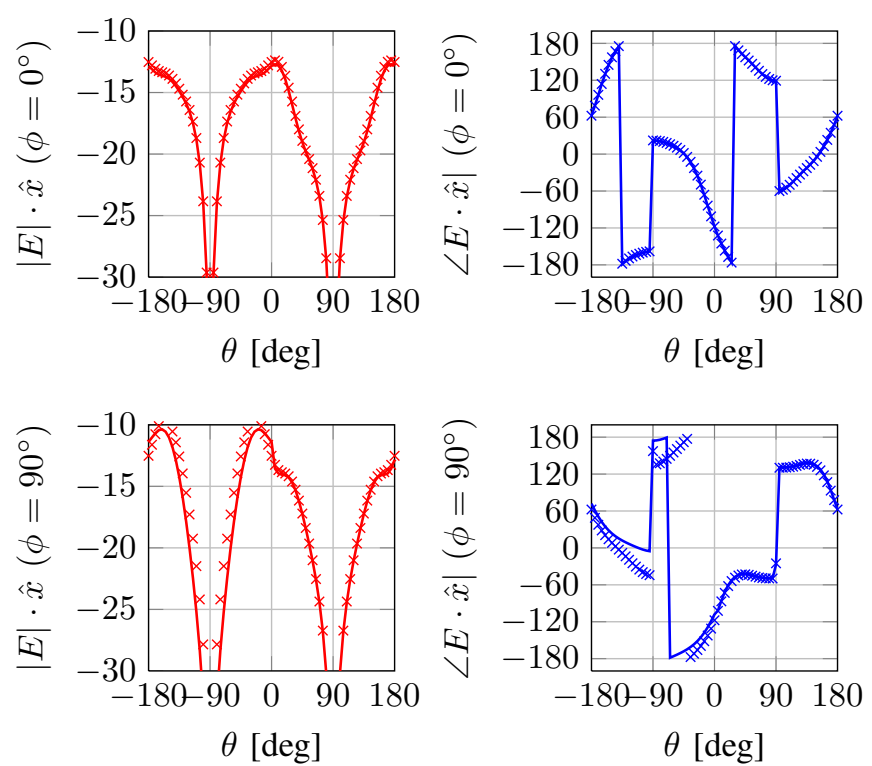

\section{- Mirrored $\times$ Computed}

\section{- Mirrored $\times$ Computed}

Fig. 6. Comparisons between mirrored and directly computed beam pattern cuts for port 3 of four dipoles arranged in an identical port layout as shown in Fig. 4, simulated at $5 \mathrm{GHz}$
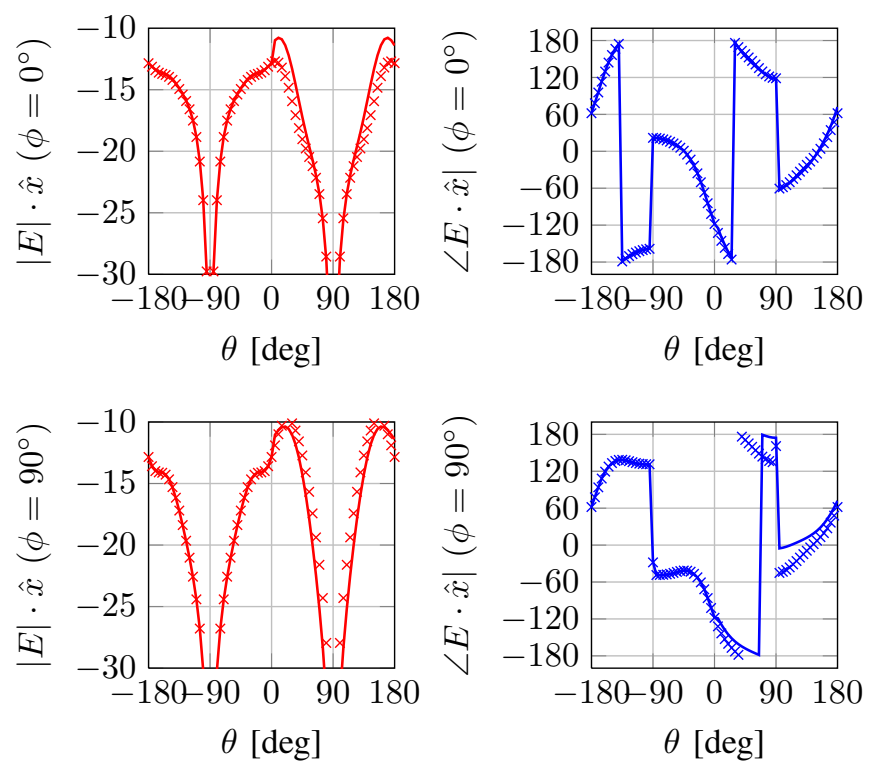

\section{- Mirrored $\times$ Computed}

\section{- Mirrored $\times$ Computed}

Fig. 7. Comparisons between mirrored and directly computed beam pattern cuts for port 4 of four folded dipoles arranged in an identical port layout as shown in Fig. 4, simulated at $5 \mathrm{GHz}$
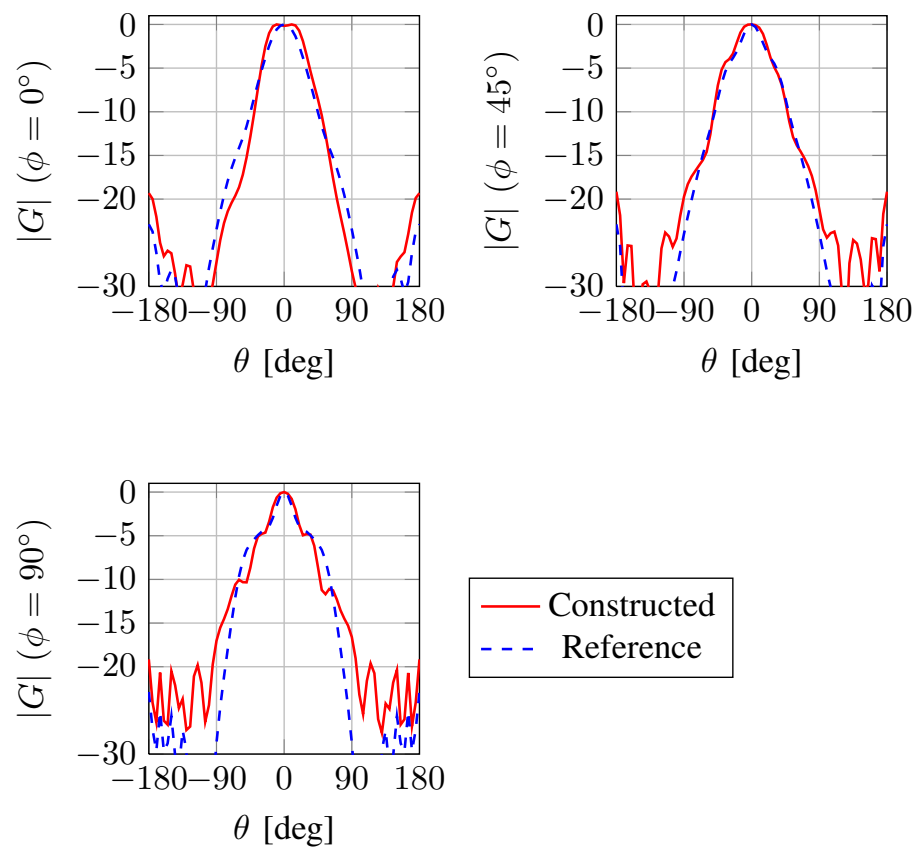

Fig. 8. The Eleven feed pattern cuts at $6 \mathrm{GHz}$, as obtained by simulating the entire antenna structure including the four folded dipoles, descrambling board and the power combining network (shown in Fig. 4), and by simulating the eight part of the antenna with the following-up procedure for reconstructing the patterns of the other ports (see Figs. 5, 6 and 7).

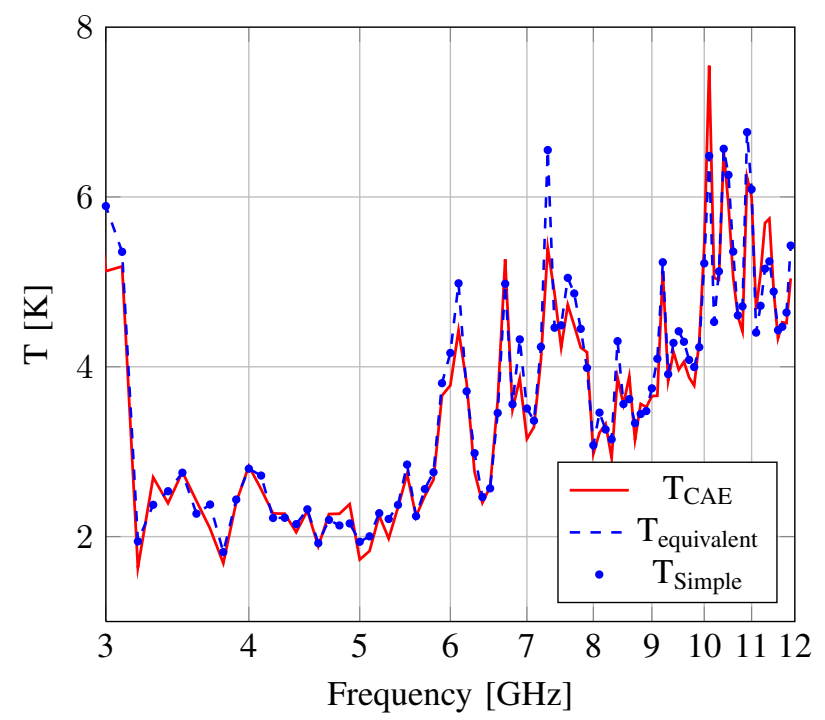

Fig. 9. Noise temperature contributions due to the LNAs and antenna impedance noise mismatch, as obtained with the complete Eleven feed receiver model (see Fig. 4) and two simpler models: equivalent model assuming no isolation and reflection losses in the LNAs, hybrids and combiner and simple model assuming, additional to the equivalent model's assumptions, identical beamformer channels and LNAs. 


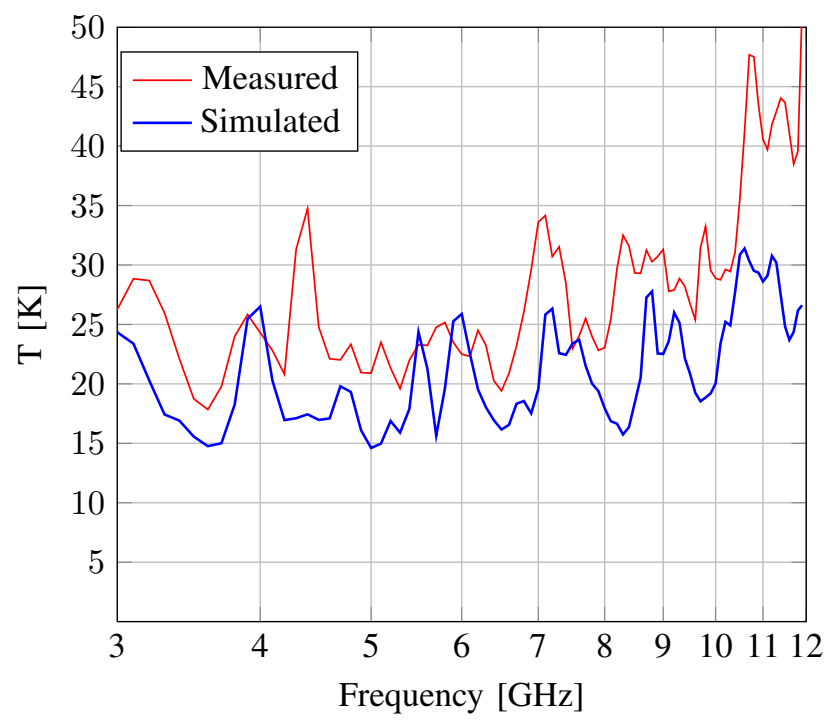

Fig. 10. Predicted and measured system noise temperatures of the Eleven feed receiver for one polarization, due to both the external and internal noise sources, including the sky background noise $\left(7 \mathrm{~K}\right.$ for $\left.\theta=0-75^{\circ}\right)$, thermal ground noise $\left(300 \mathrm{~K}\right.$ for $\left.\theta=75-180^{\circ}\right)$, antenna ohmic loss (at physical temperature of $25 \mathrm{~K}$ ), noisy LNAs and power combining network.

the feed, LNA noise sources, coupling between the dipole elements, reflections between all the beamforming elements, including the LNAs. The predicted and measured system noise temperatures are plotted in Fig. 10. All the measurements where done at Onsala Space Observatory, with the feed placed looking skyward and without a reflector. This setup increases the measured noise, as it increases the measured noise temperature, compared to a set including the reflector antenna, due to the additional ground noise pick-up component in the directions $\theta=90-180^{\circ}$. Both the measured and simulated data are smoothed using a moving average over $200 \mathrm{MHz}$ in order to reduce random measurement noise. The model underestimates the measured noise by $7.3 \mathrm{~K}$ averaged across the band, this underestimate is mainly due to using a uniform sky brightness temperature distribution, where in reality it increase at the higher end of the band, as can be seen in Fig. 10. This can be accounted for using a better atmospheric model but here a standard model is preferred. The estimated thermal noise component of the antenna due to the radiation efficiency loss $\left(\eta_{\mathrm{rad}}\right)$ is added onto to the CAESAR prediction based on measurements done of the Eleven antenna in the spherical near-field test range at DTU (Technical University of Denmark) [7]. Adding $\eta_{\text {rad }}$ post modelling increases the prediction fractionally, $\eta_{\mathrm{rad}}$ is simulated close to 0.9 so the effect is small, but should (and in future work will) be added as part of the simulated CAESAR model. The differences seen are considered accounted for or within the noise of the measurement.

\section{CONCLUSION}

The characterization of low-noise multi-port wideband receiving systems is a challenging problem requiring advanced modelling methods and measurement techniques. A system model has been developed in CAESAR incorporating data from CST, by developing a mathematically general procedure to transform the antenna radiation and impedance characteristics between the CST and CAESAR software. This method and model has been validated for a simple system of folded dipoles and used with the Eleven feed. The resonance structure of the system noise temperature reproduces well the measurements performed with the actual UWB LNAs, with differences between the simulations and measurements of $7 \mathrm{~K}$ on average over the frequency band. The model works well for predicting the resonance behaviour of the system. Future work on the model is focused on using a more accurate sky temperature distribution and beam patterns, which include the effect of the cooling cryostat's ring (which might produce ground reflections) and radome's window (which adds loss).

\section{ACKNOWLEDGEMENT}

This work was done with support from the South African National Research Foundation (NRF), Swedish Research Council (SIDA), and VINNOVA and NWO Rubicon grants.

\section{REFERENCES}

[1] P. Hall, The SKA: An Engineering Perspective. Singer, 2005.

[2] A. Whitney, A. Niell, B. Corey, H. Hase, E. Himwich, H. Hinteregger, T. Kondo, Y. Koyama, C. Ma, Z. Malkin, B. Petrachenko, W. Schlueter, H. Schuh, D. Shaffer, G. Tuccari, and N. Vandenberg, "VLBI2010: Current and future requirements for geodetic VLBI systems, IVS WG3 report," IVS WG3 Report, http://ivscc.gsfc.nasa.gov/about/wg/wg3/, Tech. Rep., 2005.

[3] K. F. Warnick, M. V. Ivashina, and S. G. Hay, "Guest editorial for the special issue on antennas for next generation radio telescopes," Antennas and Propagation, IEEE Transactions on, vol. 59, no. 6, pp. 1786 - 1789, June 2011.

[4] R. Maaskant, "Analysis of large antenna systems," Ph.D. dissertation, Eindhoven Univ. of Technology, The Netherlands, 2010.

[5] M. V. Ivashina, R. Maaskant, and B. Woestenburg, "Equivalent system representation to model the beam sensitivity of receiving antenna arrays," IEEE Antennas and Wireless Propogation Letters, vol. 7, pp. 733-737, 2008.

[6] M. Ivashina, O. Iupikov, R. Maaskant, W. van Cappellen, and T. Oosterloo, "An optimal beamforming strategy for wide-field surveys with phased-array-fed reflector antennas," Antennas and Propagation, IEEE Transactions on, vol. 59, no. 6, pp. $1864-1875$, June 2011.

[7] J. Yang, M. Pantaleev, P.-S. Kildal, B. Klein, Y. Karandikar, L. Helldner, N. Wadefalk, and C. a. Beaudoin, "Cryogenic 2-13 GHz eleven feed for reflector antennas in future wideband radio telescopes," Antennas and Propagation, IEEE Transactions on, vol. 59, no. 6, pp. 1918 - 1934, June 2011.

[8] J. Yang and P.-S. Kildal, "Optimization of reflection coefficient of large log-periodic array by computing only a small part of it," Antennas and Propagation, IEEE Transactions on, vol. 59, no. 6, pp. 1790 - 1797, June 2011.

[9] M. W. Pospieszalski, "Modeling of noise paramaters of MESFETs and MODFETs and their frequency and temperature dependence," IEEE Transactions on Microwave Theory and Techniques, vol. 9, pp. 13401349, September 1989. 